\title{
Impact of rumors or misinformation on coronavirus disease (COVID-19) in social media
}

\author{
Samia Tasnim, ${ }^{1}$ Md Mahbub Hossain, ${ }^{1}$ Hoimonty Mazumder ${ }^{2}$
}

\begin{abstract}
The COVID-19 pandemic has not only caused significant challenges for health system all over the globe but also fueled the surge of numerous rumors, hoaxes and misinformation, regarding etiology, outcomes, prevention, and cure of the disease. This misinformation are masking healthy behaviors and promoting erroneous practices that increase the spread of the virus and ultimately result in poor physical and mental health outcomes among individuals. Myriad incidents of mishaps caused by these rumors was reported across the world. To address this issue the frontline healthcare providers should be equipped with the most recent research findings and accurate information. The mass media, health care organization, community-based organizations, and other important stakeholders should build strategic partnerships and launch common platforms in disseminating authentic public health messages. Advanced technologies like natural language processing or data mining approaches should be applied in detection and removal online content with no scientific basis from all social media platforms. Those involved with the spread of such rumors should be brought to justice. Telemedicine based care should be established at a large scale to prevent depletion of limited resources.

Keywords: COVID-19, Coronavirus, Health Communication, Social Media, Health Policy
\end{abstract}

\footnotetext{
${ }^{1}$ School of Public Health, Texas A\&M University, College Station, TX 77843, USA.

2 IPAS, Dhaka, Bangladesh Corresponding Author: Dr. Samia Tasnim MD, School of Public Health, Texas A\&M University, College Station, TX 77843, USA. Email: tasnim@tamu.edu
} 


\section{Coronavirus disease (COVID-19) and flow of (mis)information in social media}

The coronavirus disease 2019 (COVID-19) is a respiratory tract illness resulting from Severe Acute Respiratory Syndrome Coronavirus 2 (SARS-CoV-2) infection, which has spread all over the globe, making it a major public health challenge across health systems. Simultaneously, numerous rumors, misinformation, and hoaxes appeared on several social media platforms regarding the etiology, outcomes, prevention, and cure of the disease ${ }^{1}$. The pressing issue is fake news spread more rapidly in social media than the ones from reliable sources and damages the authenticity balance of news ecosystem.

A recent analysis showed that during the 2016 presidential election in the United States, fake and biased news were more outspread than news from 19 authentic sources combined ${ }^{2}$. It is becoming a public health concern as the exposure to high-volume of information can lead to media fatigue causing relaxation of healthy behaviors essential to protect individuals. Also, misinformation and rumors regarding COVID-19 are masking healthy behaviors (such as hand washing, social distancing etc.) and promoting erroneous practices that increase the spread of the virus and ultimately result in poor physical and mental health outcomes among individuals. For example, in India, a father of three was reported to commit suicide upon hearing his diagnosis of COVID-19³.

Another example of hazards attributable to improper health communication can be drawn from Nigeria, where the health officials found several cases of overdose of Chloroquine (a drug used to treat malarial parasite) after the news regarding the effectiveness of the drug for treating COVID19 spawned in the news media. ${ }^{4}$ Another rumor about the national lockdown in the United States has fueled panic buying of groceries and stationeries, which disrupted the supply chain, and exacerbated demand-supply gaps and food insecurities among individuals with low socioeconomic 
status and other vulnerable populations ${ }^{5}$. A similar phenomenon was observed in other economies around the world, which may have prolonged impacts on public health nutrition and psychosocial health among the affected populations.

Many misinformation related to diagnosis and treatment of COVID-19 has confused the general population as well as the health care providers, who are battling a relatively less researched disease. This is also reducing the legitimacy of new scientific discovery regarding the cure or vaccine for this disease. These hoaxes and rumors are also creating a social stigma around this infection, which has resulted in reduced compliance with home quarantine and social isolation. Several countries have reported incidents where hundreds of individuals were infected from a single person who visited their mosque/church despite their doctor's advice to remain isolated at home ${ }^{6}$. Such problems are contributing to suboptimal control of COVID-19 pandemic across populations.

\section{Potential strategies to ensure effective health communication on COVID-19}

It is understandable that people living in quarantine, isolation, or at risk of infectious disease outbreak are likely to experience psychosocial stress and adverse health outcomes, which may evoke interests in learning more about the disease ${ }^{7}$. However, such situations require assurance complemented by flow of correct information. This need is acknowledged by the World Health Organization (WHO), which has partnered with several social media and seven major tech companies namely Facebook, Google, LinkedIn, Microsoft, Reddit, Twitter, and YouTube, and agreed to stump out the frauds and misinformation, and promote critical updates from healthcare agencies ${ }^{8}$. 
Despite the agreements, the rumors are still rampant all over the platforms, which necessitates several multipronged interventions to address the same. First, the frontline healthcare providers should be equipped with the most recent research findings and accurate information, which can be used in direct caregiving and communicated with the patients or populations at risk. This may also leverage more robust patient-provider communication and result in better health outcomes. Previously integration of information technology successfully improved patient care in managing mass casualty incidents. This brought several positive outcomes in the form of optimization of resources, an extension of care and improved health outcomes of patients. ${ }^{9}$

Secondly, mass media, community organizations, support groups, and civil society may play critical roles in disseminating authentic information. To achieve this, it is necessary to build strategic partnerships at local and global levels, connecting offline and online resources in a coordinated manner so that validated information is communicated across platforms.

Third, all hoaxes and rumors should be removed from all online platforms, and only scientifically sound information should be allowed to increase awareness among mass people. Advanced technologies like natural language processing or text-mining approaches may be helpful in detecting online content that has no scientific basis. The latest data-mining algorithms are successfully detecting the unique characteristics of all kinds of fake news and removing them from their respective platforms ${ }^{2}$. Twitter, Reddit, and Amazon have already implemented such methods to remove fake accounts or product reviews. Application of the same principals in removing all rumors and hoaxes regarding this pandemic despite its unique challenges is evidently feasible. Therefore, social media and other online providers should adopt such measures to identify and eliminate potentially harmful misinformation and rumors. 
Fourth, online portals and personnel involved with the production and propagation of such misinformation should be brought to justice. Local and national regulatory authorities and law enforcement agencies should be made aware of these challenges to address these challenges in respective contexts comprehensively. In this regard, governments of many countries have arrested individuals involved with the spread of such rumors. ${ }^{10}$ However, before enforcing any of these laws worldwide, authorities should conduct a proper investigation and ensure the legal rights of the convicts.

Fifth, common platforms should be launched providing precautionary information in both online and offline media on a large scale. Successful strategies for mass media campaigns from the last few epidemics should be identified and implemented. These messages should be culturally tailored and translated to several languages to increase outreach. The media coverage of the recent SARS, H1N1, MERS-CoV, Ebola, and H7N9 epidemics had a significant effect on reducing the total spread of these pathogens by promoting healthy behaviors. Factual information regarding the COVID-19 is also expected to have similar results.

Sixth, using telemedicine approach evidence-based care and information services should be made available for people with limited access to care such as people in the rural areas, elderly population, or simply stuck at home due to lockdown. This will not only decrease the chances of the hospitalacquired infection but also reduce the utilization of resources like ventilators, personal protective equipment for doctors etc.

Lastly, transparency, good information governance, and leadership across communities and institutions should be promoted to remove any misleading information related to COVID-19, 
which are essential for the greater good of populations experiencing health and psychosocial burden of this pandemic.

Acknowledgement: None.

Conflicts of interest: We declare no conflicts of interest.

Funding: No funding was received at any stage of preparing this manuscript

\section{References:}

1. Raman Sandhya. Public health experts worry about spread of COVID-19 misinformation.https://www.rollcall.com/2020/03/18/public-health-experts-worry-aboutspread-of-covid-19-misinformation/. Published 2020.

2. Shu K, Sliva A, Wang S, Tang J, Liu H. Fake News Detection on Social Media. ACM SIGKDD Explor Newsl. 2017. doi:10.1145/3137597.3137600

3. Joe W. Coronavirus: Indian man "died by suicide" after becoming convinced he was infected. The Telegraph. https://www.telegraph.co.uk/global-health/science-anddisease/coronavirus-indian-man-died-suicide-becoming-convinced-infected/. Published 2020.

4. Busari S, Adebayo B. Nigeria records chloroquine poisoning after Trump endorses it for coronavirus treatment. $C N N$. https://www.cnn.com/2020/03/23/africa/chloroquine-trump- 
nigeria-intl/index.html. Published 2020.

5. Spencer SH. False Claims of Nationwide Lockdown for COVID19.https://www.factcheck.org/2020/03/false-claims-of-nationwide-lockdown-for-covid$19 /$.

6. Kasulis K. "Patient 31" and South Korea's sudden spike in coronavirus cases. Aljazeera. https://www.aljazeera.com/news/2020/03/31-south-korea-sudden-spike-coronaviruscases-200303065953841.html.

7. Hossain MM, Sultana A, Purihit N. Mental health outcomes of quarantine and isolation for infection prevention: A systematic umbrella review of the global evidence. 2020. https://doi.org/10.31234/osf.io/dz5v2.

8. Major tech platforms say they're 'jointly combating fraud and misinformation' about COVID-19. The verge. https://www.theverge.com/2020/3/16/21182726/coronaviruscovid-19-facebook-google-twitter-youtube-joint-effort-misinformation-fraud.

9. Zhao X, Rafiq A, Hummel R, Fei DY, Merrell RC. Integration of information technology, wireless networks, and personal digital assistants for triage and casualty. Telemed $\mathrm{J} e$ Health. 2006. doi:10.1089/tmj.2006.12.466

10. NHK police arrest man "for spreading fake virus news."https://news.rthk.hk/rthk/en/component/k2/1506616-20200204.htm. 
